\title{
Photoionization Microscopy for a Hydrogen Atom in Parallel Electric and Magnetic Fields
}

\author{
M. Deng \\ W. Gao \\ Rong Lu \\ L. You \\ John B. Delos \\ William \& Mary
}

Follow this and additional works at: https://scholarworks.wm.edu/aspubs

\section{Recommended Citation}

Deng, M.; Gao, W.; Lu, Rong; You, L.; and Delos, John B., Photoionization Microscopy for a Hydrogen Atom in Parallel Electric and Magnetic Fields (2016).

https://doi.org/10.1103/PhysRevA.93.063411

This Article is brought to you for free and open access by the Arts and Sciences at W\&M ScholarWorks. It has been accepted for inclusion in Arts \& Sciences Articles by an authorized administrator of W\&M ScholarWorks. For more information, please contact scholarworks@wm.edu. 


\title{
Photoionization microscopy for hydrogen atom in parallel electric and magnetic fields
}

\author{
M. Deng, ${ }^{1}$ W. Gao ${ }^{2,3}$ Rong Lu, ${ }^{1}$ J. B. Delos,${ }^{4}$ L. You, ${ }^{1}$ and H. P. Liu ${ }^{* 2}$ \\ ${ }^{1}$ State Key Laboratory of Low-Dimensional Quantum Physics, \\ Department of Physics, Tsinghua University, Beijing 100084, China \\ ${ }^{2}$ State Key Laboratory of Magnetic Resonance and Atomic and Molecular Physics, \\ Wuhan Institute of Physics and Mathematics, \\ Chinese Academy of Sciences, Wuhan 430071, Peoples Republic of China \\ ${ }^{3}$ Graduate School of the Chinese Academy of Sciences, Beijing 100049, People's Republic of China \\ ${ }^{4}$ College of William and Mary, Williamsburg, Virginia 23187-8795, USA
}

\begin{abstract}
In photoionization microscopy experiments, an atom is placed in static external fields, it is ionized by a laser, and the electron falls onto a position-sensitive detector. The current of electrons arriving at various points on the detector depends upon the initial state of the atom, upon the excited states to which the electron is resonantly or non-resonantly excited, and upon the various paths leading from the atom to the final point on the detector. We report here the first quantum-mechanical computations of photoionization microscopy in parallel electric and magnetic fields. We focus especially on the patterns resulting from resonant excited states. We show that the magnetic field substantially modifies some of these resonant states, confining them in the radial direction, and that it has a strong effect on the interference pattern at the detector.
\end{abstract}

PACS numbers: $32.80 . \mathrm{Fb}, 07.81 .+\mathrm{a}, 32.60 .+\mathrm{i}$

\section{INTRODUCTION}

Recent developments in the field of photoelectron imaging have allowed the direct observation of the oscillatory structure of a microscopic wave-function on a macroscopic scale [1-4]. The theory of photodetachment or photoionization microscopy was introduced in the early 1980s [5-7], with a proposed experiment in which an electron of a fixed energy is detached from a negative ion or ionized from an atom in an applied static field, and then accelerated by the field to a position-sensitive detector. The outgoing electrons can display circular interference rings on this detector. The observed patterns result from interferences of electron waves traveling along different classical paths from the ion or atom to the detection point. For photodetachment, only two trajectories interfere at each detector position and the observed structures can easily be interpreted based on analytical expressions for the accumulated phases along both trajectories [8-10]. However, in photoionization, because of the long-range Coulomb interaction, the trajectories are more complex, and an infinite number of classical trajectories arrives at any point in the classically allowed region on the detector.

Up to now, experimental measurements of photoionization microscopy have been carried out only in a pure electric field (no magnetic field). The first experimental implementation was for Xe atom by Nicole et al. [2], following the pioneering study on slow photoelectron imaging [11]. The interference pattern was found to evolve smoothly with the excess energy above the saddle point and was only weakly affected by the presence of Stark res-

*E-mail: liuhongping@wipm.ac.cn onances. The observed interference patterns were qualitatively explained by Bordas et al. [9], using a semiclassical framework. Subsequent studies with Xe atom [12] investigated the influence of Stark resonances, and concluded that the semiclassical theory applied to Stark hydrogen atom was unable to assign all observed resonances.

On the theoretical side, pioneering work on dynamics of electrons photoionized from rubidium in a pure electric field was carried out by Robicheaux and Shaw [13] using a form of Harmin's frame-transformation theory [14]. A quantum theory for photoelectron microscopy in both hydrogen and lithium atoms was developed by Zhao et al. $[15,16]$. They found that Stark resonances dramatically change the electron spatial distribution. Recently, such theoretical predictions for the effects of resonances $[10,16]$ were confirmed by measurements on hydrogen [3] and lithium [4]. In contrast to observations with Xe atoms, microscopy images of $\mathrm{Li}$ and $\mathrm{H}$ atoms are found to be sensitive to the presence of resonances.

A natural question to ask concerns how the microscopy interference patterns change if a magnetic field is added parallel to the electric field (Fig. 1). For the case of photodetachment, where the Coulomb field is absent, and trajectories only spiral around magnetic field lines while falling at constant acceleration, there have been a number of recent studies [17-20]. For photoionization, however, again the Coulomb interaction dramatically complicates the situation, as infinite sets of electron trajectories from different families, some of which are chaotic, contribute to the observed electron spatial density distribution [21].

The spectrum of hydrogen atom in parallel fields has been studied by many researchers at the energy far below the Stark saddle point [22-24]. To our knowledge, however, for the photoionization microscopy of atom in parallel fields, there has been no research report except our 


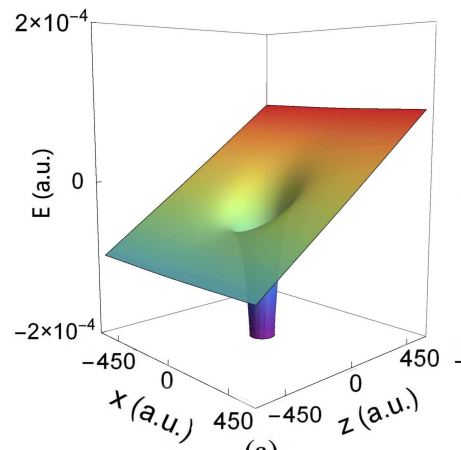

(a)

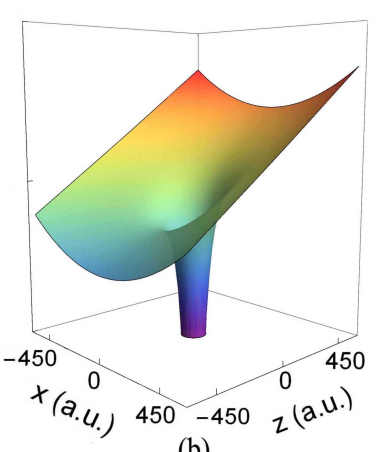

(b)
FIG. 1: (Color online) The potential energy surfaces $(E)$ of hydrogen atom in a uniform electric field $(F=808 \mathrm{~V} / \mathrm{cm})$ along $z$-axis in (a), compared to the case in parallel electric and magnetic fields $(F=808 \mathrm{~V} / \mathrm{cm}, \mathrm{B}=8 \mathrm{~T})$ in (b). The singular attractive feature at the origin comes from the Coulomb interaction.

previous semiclassical open-orbit theory approach [21], where the tunneling and resonance effects were omitted. Two related aspects of that work show the need for the present study. (1) Trajectories were started at the nucleus, and were assumed to be isotropically distributed. (2) Effects of resonances were ignored; thus the energy domain of interest was approximated as an unstructured continuum [9]. However, as already mentioned, it is known that for hydrogen in a pure electric field, resonances have a large effect [16], greatly changing the outgoing waves in narrow ranges of energy. A semiclassical open-orbit theory of photionization microscopy including effects of resonances is currently unavailable.

Accordingly, in this paper we calculate by quantum theory the patterns that may be seen in photoionization microscopy experiments on hydrogen in parallel electric and magnetic fields, giving particular attention to the effects of resonances. There are two parts to our calculations. (1) We find the energies of resonances in parallel fields using the complex-rotation technique (CRT) [25]. (2) Using a wavepacket propagation method, we compute the wave function extending to large distances, and show how the patterns on the detector change when a parallel magnetic field is applied.

\section{THEORY AND NUMERICAL APPROACH}

Atomic units are used throughout this work unless otherwise specified. The Hamiltonian for our model of a neutral hydrogen atom in uniform parallel electric and magnetic fields $\boldsymbol{F}=F e_{z}$ and $\boldsymbol{B}=B e_{z}$ is expressed in spherical coordinates as follows

$$
H=\frac{p^{2}}{2}-\frac{1}{r}+F r \cos \theta+\frac{B}{2} L_{z}+\frac{B^{2}}{8} r^{2} \sin ^{2} \theta,
$$

where $F, B$ are the electric and magnetic field strength, respectively. The first term on the rhs of the above
Eq. (1) is the kinetic energy for the electron, the second term denotes its Coulomb potential, the third term corresponds to the Stark interaction of the electron with the applied electric field, while the last two terms are respectively the paramagnetic and diamagnetic interactions from the external magnetic field. In the parallel field configuration as we study, the above model Hamiltonian preserves azimuthal symmetry, which ensures the $z$-component of the angular momentum $L_{z}$ is the constant of motion and the magnetic quantum number $m$ a good quantum number. We take $m=0$ in all reported calculations here in order to match the experimental condition of Ref. [3] where the laser pulse is linearly polarized along the direction of electric and magnetic fields.

In a uniform electric field, electron Stark states are conveniently treated in separable parabolic coordinates $\eta$ and $\xi$. The electron motion is bounded in $\xi=r+z$ and unbounded in $\eta=r-z$. The Stark manifolds are denoted by $\left(n_{1}, n_{2}, m\right)$ in terms of parabolic quantum numbers $n_{1}$ and $n_{2}\left(n=n_{1}+n_{2}+|m|+1\right)$, which respectively characterize the numbers of wave-function nodes along the $\xi$ and $\eta$ coordinates. Red Stark states $\left(n_{1}<n_{2}\right)$ are localized on the downhill side of the Coulomb + pure electric field potential and they are more weakly bounded in their spatial distributions than blue Stark states $\left(n_{1}>n_{2}\right)$ on the opposite uphill side. When the quantum number $n_{1}$ of a red state is equal to the $n_{2}$ of a blue state, these two states will have the same transverse nodal structure, except that the nodes for the former cross the negative $z$ axis while for the latter they cross the positive $z$-axis. As long as the Stark nodal structure (the number of nodes in parabolic coordinates $\eta$ and $\xi$ ) remains intact when the magnetic field is introduced in addition to the electric field, we can still identify the Stark resonances in parallel fields by effective quantum numbers $\left(\tilde{n}_{1}, \tilde{n}_{2}, m\right)$ in reference to the Stark state notation $\left(n_{1}, n_{2}, m\right)$ in a pure electric field.

\section{A. Resonances in parallel fields}

The method of CRT can be found in detail in Ref. [25]. Upon rotating the radial variable $r$ by an angle $\alpha$ in the complex plane according to $r=|r| e^{i \alpha}$, we transform the Schrödinger equation into a complex general eigenvalue problem,

$$
H C=E S C,
$$

where $E$ and $C$ represent the complex eigenvalues and their corresponding eigenvectors, and $S$ denotes the overlap matrix. A Lanczos algorithm for the general complex eigen-problem is then applied to solve for the eigenvalue $E$ and eigenvector $C$. If $\alpha$ is sufficiently large, resonance states become square-integrable states. When $\alpha$ is reduced, a long tail in the wave-function of the resonance state appears. Also other quasi-states appear which are unstable under variation of $\alpha$. Because we want to display the long tails of the resonant states, we show graphs 


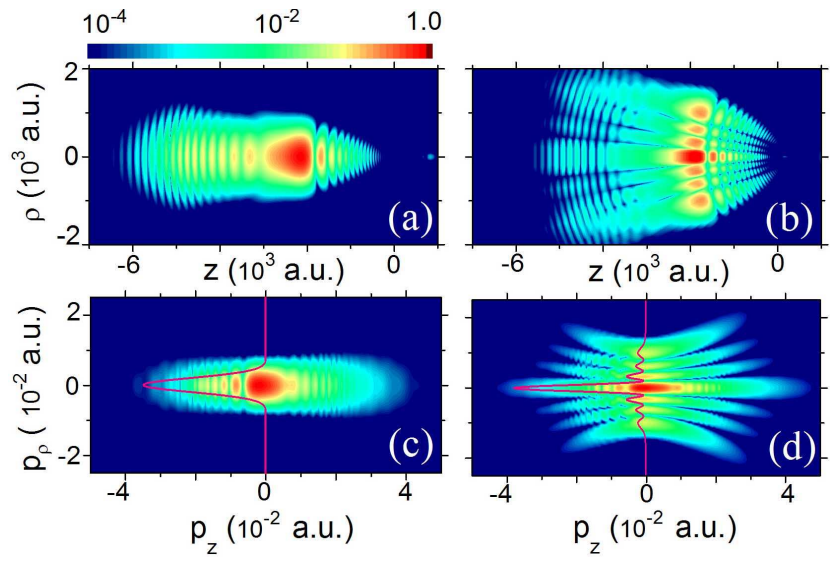

FIG. 2: (Color online) The quantum theory calculated 2D electron spatial and momentum distributions in a uniform pure electric field $F=808 \mathrm{~V} / \mathrm{cm}$ for certain resonant states. (a) and (b) are spatial distributions for the two red states $(0,29,0)$ and $(3,26,0)$, respectively. A tail of the spatial distribution is obviously noticeable beyond the saddle point $z=-1 / \sqrt{F}=-2522.72$ a.u. in both (a) and (b). The corresponding $2 \mathrm{D}$ momentum distributions are shown in (c)-(d). In addition, 1D momentum distributions along $p_{\rho}$ obtained after integrating over $p_{z}$ are also shown by pink curves. The same number $\tilde{n}_{1}$ of nodes as in their corresponding spatial distributions can be clearly counted.

of stable resonant states of sufficiently small values of $\alpha=0.01$ whose tails are visible. At these resonant energies, ionization is strongly enhanced, and the distributions of both electron spatial probability density and its two-dimensional (2D) momentum are highly structured. For example, we take two red Stark states as shown in Fig. 2, both having $\tilde{n}_{1}$ nodes in their transverse structure as carefully illustrated in Figs. 2(a)-(b), and their one dimensional momentum distributions of $p_{\rho}$ are characterized by the same node number as well in Fig. 2(c)(d). From Fig. 2, we can see that for red states with smaller quantum numbers $\tilde{n}_{1}$, their spatial distributions and the momentum distributions are concentrated more along the central symmetrical axis. The blue resonances with smaller quantum number $\tilde{n}_{2}$ show similar features. These resonance states are found to be reasonably immune to the introduction of external magnetic field, unlike the states with large spatial distributions perpendicular to the $z$-axis or wide momentum distributions in the $p_{\rho}$ direction. We will discuss this observation in more detail later.

\section{B. Wavepacket propagation in parallel fields}

To describe the wavepacket propagation dynamics in parallel fields, we follow the approach of Ref. [3], except that we solve the time dependent Schrödinger equation using a split-operator technique instead. The wavefunction $\psi(r, t)$ at any time $t$ obeys the time-dependent
Schrödinger equation

$$
i \frac{\partial}{\partial t} \Psi(r, t)=\left(H_{A}+H_{F B}+H_{L}\right) \Psi(r, t)
$$

with

$$
\begin{aligned}
H_{A} & =\frac{p^{2}}{2}-\frac{1}{r} \\
H_{F B} & =F r \cos \theta+\frac{B}{2} L_{z}+\frac{B^{2}}{8} r^{2} \sin ^{2} \theta, \\
H_{L} & =F_{L}(t) r \cos \theta,
\end{aligned}
$$

respectively denoting the free-field hydrogen atom Hamiltonian, the interaction between the atomic electron and the external parallel fields, and with the pumping laser pulse characterized by $F_{L}(t)$. Here the spin-orbit interaction is omitted since it is very small for high Rydberg states. The spin-orbit interaction is proportional to $1 / r^{3}$ and decreases quickly at large $r$. The paramagnetic term $\frac{B}{2} L_{z}=\frac{B}{2} m$ is a constant for a given state with magnetic quantum number $m$, in our case, $m=0$. As the paramagnetic term is not concerned with the radius, even considering the spin, it only contributes a constant energy shift.

To the first order approximation, the wave-function describing the excitation from an initial state $\Phi(r)$ at energy $E_{0}$ to a final state $\Psi(r, t)$ of energy $E$ is given by

$$
\Psi(r, t)=\Phi(r) e^{-i E_{0} t}+\psi(r, t) e^{-i E t},
$$

where the first term satisfies the free-field Schrödinger Eq. (3) with only $H_{A}$ included. Substituting Eq. (7) into Eq. (3) leads to an inhomogeneous Schrödinger equation for $\psi(r, t)$

$$
i \frac{\partial}{\partial t} \psi(r, t)-\left(H_{A}+H_{F B}-E\right) \psi(r, t)=S(r, t) .
$$

The excitation process is taken into account via the source term $S(r, t)$ defined as

$$
S(r, t)=e^{-\frac{t^{2}}{(\Delta \tau)^{2}}} r S(r) \cos \theta Y_{l}^{m},
$$

with $Y_{l}^{m}$ the spherical harmonic describing the initial state characterized by quantum numbers $l$ and $m$. $S(r)$ describes the radial dependence of the initial wavefunction. The laser pulse is assumed to take a Gaussian shape parameterized by a temporal width $\Delta \tau$.

By integrating Eq. (8) using the generalized pseudospectral and split-operator technique, we obtain the time-dependent wave-function numerically (see the supplementary material of Ref. [3]). In the calculation, the outer boundary of the grid of points used for the wavepacket propagation extends far past the hypothetical location of the detector ( 8000 a.u.), to avoid the reflections from the boundary. For the reported experimental conditions, the total propagation time extends to at least 500 ps. 

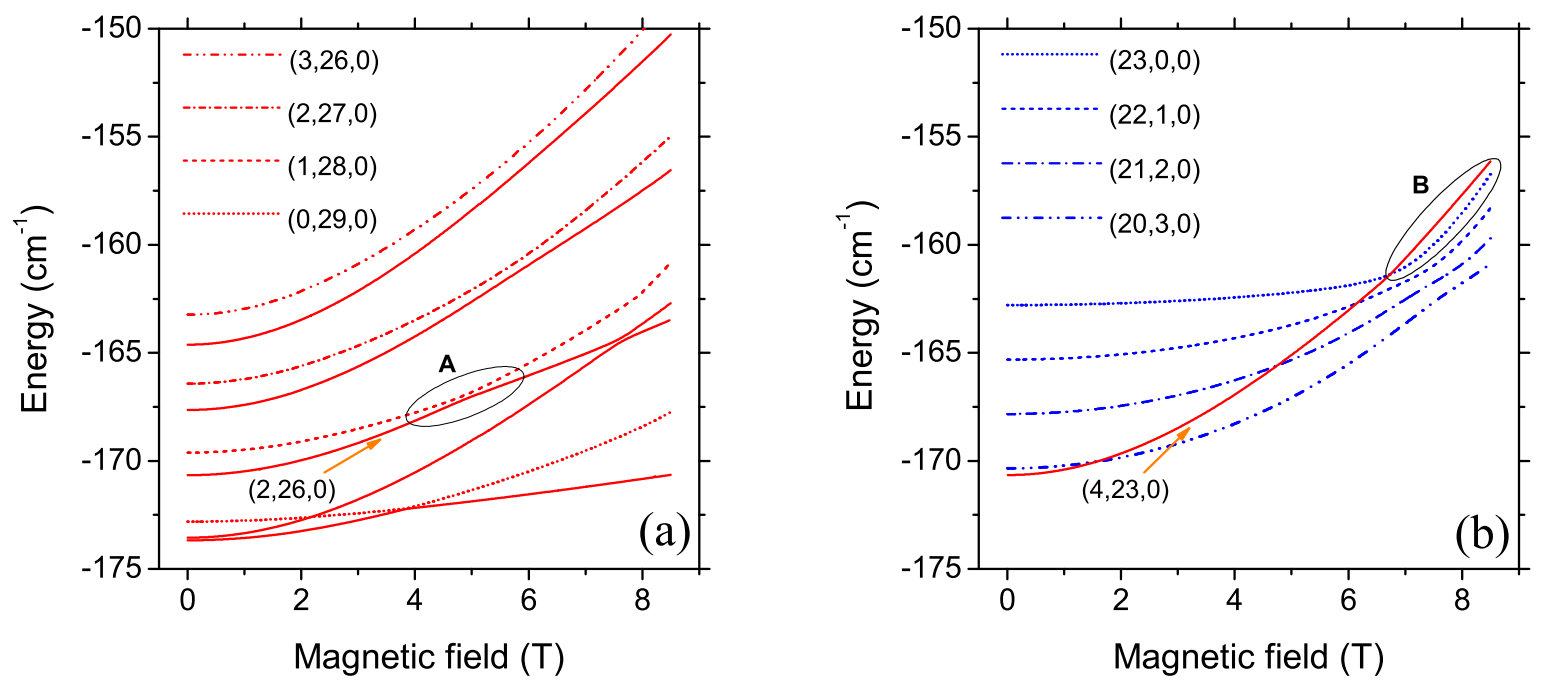

FIG. 3: (Color online) Calculated energy level maps for both red (a) and blue (b) resonances in Table I. Comparing red states with blue states having the same number of transverse nodes (0-3) it can be seen that the magnetic field has a greater effect on the energies of the red states. As a result, anticrossings occur more easily between red states (a) than between blue states (b). As an example, in region A, the anticrossing occurring between the states $(1,28,0)$ (red short dash line) and $(2,26,0)$ (red solid line) is marked. Blue states are strongly mixed with nearby red states at high magnetic field. The region B shows the blue state $(23,0,0)$ (blue short dot line) is mixed with the red state $(4,23,0)$ (red solid line).

If an imaging detector is placed at $z=z_{0}$, it will see the propagated electronic wavepacket that arrives at its surface. The generated electric signal on the detector surface is proportional to the probability flux integrated over a given propagation time $T, P\left(z_{0}\right)=\int_{t=0}^{T} d t \int_{\rho} \rho d \rho \vec{j}\left(\rho, z_{0}\right)$. $\hat{z}=\int_{t=0}^{T} d t \int_{\rho} \rho d \rho j_{z}\left(\rho, z_{0}\right)$, where the probability density $j_{z}$ can be approximated as $\nu_{z}\left|\Psi\left(\rho, z_{0}, t\right)\right|^{2}$. Here $\nu_{z}$ is the velocity of the electron at $\left(\rho, z_{0}\right)$, and is approximately equal since the electron is accelerated greatly by the applied electric field [1]. Thus the signal at $\left(\rho, z_{0}\right)$ can be expressed as $P\left(\rho, z_{0}\right) \propto \int_{t=0}^{T} d t \rho\left|\Psi\left(\rho, z_{0}, t\right)\right|^{2}$, giving a plot in 2D polar coordinates $\left(\rho, z=z_{0}\right)$.

\section{RESULTS AND DISCUSSIONS}

We first calculate the positions of the same four red resonances $\left(\tilde{n}_{1}, \tilde{n}_{2}, m\right)=(0,29,0),(1,28,0),(2,27,0)$ and $(3,26,0)$ at an electric field strength $F=808 \mathrm{~V} / \mathrm{cm}$ as the same experimental condition in Ref. [3]. Four blue resonances $(23,0,0),(22,1,0),(21,2,0)$ and $(20,3,0)$ are also calculated in order to compare with the red ones in the same energy range as listed in Table.I. The calculated values we obtain for zero magnetic field are the same as or very close to those given in Ref. [26]. Six other red resonances are also given for their interesting interaction features with the magnetic field, which we will use as an example in later discussions.

We also calculate the energy level map for these tabulated red and blue resonances at increasing magnetic field up to $8.5 \mathrm{~T}$ as shown in Fig. 3. Typically the red states are more extended in $\rho$ than blue states with the same number of transverse nodes (compare Figs 4(a) with (c) and 4(b) with (d)). Since the diamagnetic interaction is proportional to $\rho^{2}$, the energies of these red states are more sensitive to magnetic fields than those of the corresponding blue states. From a different perspective, focusing on the $p_{\rho}$ distribution shown in Fig. 2, the resonances that are narrower in $p_{\rho}$ correspond to momentum distributions more concentrated around $p_{\rho}=0$, so these states are less influenced by the Lorentz forces produced by the magnetic field acting on the $p_{\rho}$ component.

In a pure electric field, the adjacent Stark manifolds overlap for a given value of the principal quantum number $n$ if $F>1 /\left(3 n^{5}\right)$. The blue and red states in fact cross each other without coupling in hydrogen atom. In parallel fields, however, the diamagnetic interaction from the applied magnetic field couples nearby crossing Stark states, which results in anticrossing or avoided crossing level diagrams as shown in Fig. 3(a). For example, the avoided crossing region $A$ arises from coupling between the $(1,28,0)$ (red short dash line) and $(2,26,0)$ (red solid line) states. The nodal structures for states $(1,28,0)$ and $(2,26,0)$ exchange after passing the anticrossing region. In contrast, as shown in Fig. 3(b), the blue resonance states are insensitive to the magnetic field up to quite strong magnetic fields, but the blue states are contaminated by nearby red states. For example, the red state $(4,23,0)$ gradually appears along the $-z$ direction of the blue state $(23,0,0)$ in region $B$.

On one hand, the magnetic field introduces a harmonic potential perpendicular to the $z$-axis, which consequently draws the wave-functions towards the $z$-axis. This is seen 


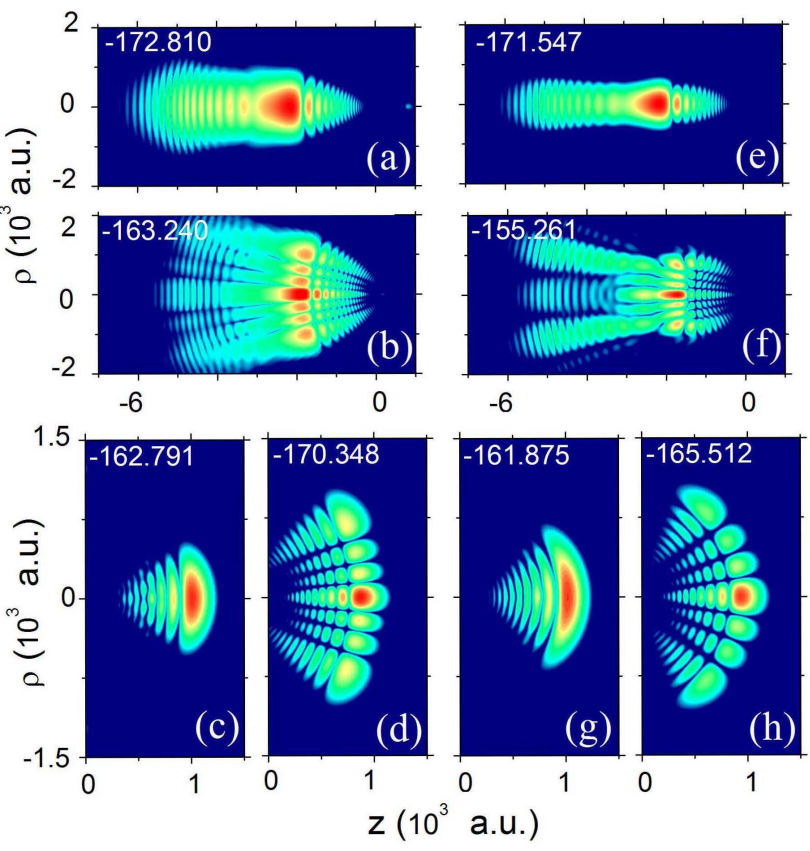

FIG. 4: (Color online) The quantum theory calculated 2D electron spatial distributions in electric field without and with parallel magnetic field applied, respectively shown in (a)-(d) and (e)-(h) for certain resonant states. The electric field has the same value $F=808 \mathrm{~V} / \mathrm{cm}$ as in Fig. 2 and the applied magnetic field is $6 \mathrm{~T}$ in $(\mathrm{e})-(\mathrm{h})$. The red resonances $((0,29,0),(3,26,0))$ are as shown in (a) and (b). Their spatial distributions are compressed in the $\rho$-direction as shown in (e) and (f) after the magnetic field is applied. However, the blue states $((23,0,0),(20,3,0))$ shown in (c)-(d) show opposite behaviors with their spatial distributions extending away from the central symmetry axis when magnetic field is applied in (g)-(h).

TABLE I: The Stark resonance positions (in $\left.\mathrm{cm}^{-1}\right)$ for hydrogen atom in a uniform electric field $(F=808 \mathrm{~V} / \mathrm{cm})$ corresponding to the conditions used for Fig. 3. As a comparison, the saddle point is located at $-174.00 \mathrm{~cm}^{-1}$ in this case.

\begin{tabular}{lclc}
\hline \hline Red States & \multicolumn{3}{l}{ Blue States } \\
\hline$\left(\tilde{n}_{1}, \tilde{n}_{2}, m\right)$ & $\mathrm{cm}^{-1}$ & $\left(\tilde{n}_{1}, \tilde{n}_{2}, m\right)$ & $\mathrm{cm}^{-1}$ \\
$(0,29,0)$ & -172.810 & $(23,0,0)$ & -162.791 \\
$(1,28,0)$ & -169.617 & $(22,1,0)$ & -165.312 \\
$(2,27,0)$ & -166.426 & $(21,2,0)$ & -167.831 \\
$(3,26,0)$ & -163.240 & $(20,3,0)$ & -170.348 \\
$(4,24,0)$ & -164.623 & & \\
$(3,25,0)$ & -167.645 & & \\
$(4,23,0)$ & -170.655 & & \\
$(2,26,0)$ & -170.662 & & \\
$(3,24,0)$ & -173.554 & & \\
$(1,27,0)$ & -173.676 & & \\
\hline \hline
\end{tabular}

clearly if we compare the two red states as shown in Figs. 4(a)-(b) in a pure electric field with Figs. 4(e)-(f) in parallel fields. On the other hand, however, the applied magnetic field also raises the overall potential surface which pushes states to higher energies. This gives rise to a nonnegligible factor when the wave-function is extended out in $\rho$ coordinate. On the contrary, for the two blue states shown in Figs. 4(c)-(d), their wave functions in parallel fields are pushed away from the central symmetry axis by the magnetic field [Figs. 4(g)-(h)]. The competition between these two factors determines the behavior of the final distribution of the wave function along the $\rho$-axis.

Because of their long lifetimes, blue resonances are not suitable for photoionization microscopy experiments. We instead examine the red resonance state $\left(\tilde{n}_{1}, \tilde{n}_{2}, m\right)=$ $(1,28,0)$ with a simple transverse nodal structure to study the characteristic features of wave propagation in parallel fields. In a pure uniform electric field, the calculated Stark state electron radial distribution on the detector is shown in Fig. 5(a), which is in good agreement with reported experimental results [3] and other theory calculations [26]. Figure 5(b)-(e) shows the same spatial distributions for resonances in parallel fields. As the magnetic field increases, the long "hair" in Fig. 5(a) changes into "braids" in Fig. 5(d) and (e), due to the focusing effect related to the spiraling of the electron around the magnetic field lines. This feature will also be explained by the classical calculation later.

In a pure electric field, the number of dark fringes of the interference patterns on the detector directly reveals the transverse node number of the Stark state (Fig. 5(a)). However, this general correspondence no longer holds true in parallel fields. The peak position and the relative strength of the radial probability distribution vary with magnetic field as shown in Fig. 5(a)-(d) for increasing magnetic field from $0 \mathrm{~T}$ to $6 \mathrm{~T}$. At $8 \mathrm{~T}$ we find only one peak remains (Fig. 5(e)). The transverse nodal structure for this red state remains clearly distinguished within the saddle point in parallel fields (Fig. 5(e)), but it does not extend to the location of the detector.

For comparison, we also carried out trajectory calculations based on the semiclassical open-orbit theory [21], within which the resulting wave-function $\psi_{z_{0}}(\rho)$ at point $M\left(\rho, z_{0}\right)$ on the detector corresponds to the sum over all possible trajectories. Several open-orbits at $8 \mathrm{~T}$ in Figs. $5(\mathrm{e})$ are presented in solid color lines which can be easily compared to analogous ones at $0 \mathrm{~T}$ in Figs. 5(a). Superimposed on their backgrounds are the electron spatial distributions from quantum calculations. Following Mitchell and Delos [27], we classify the trajectories using a string of the symbols $\{-, o\}$. The ejection angles for these orbits are selected as the extreme points labeled by the symbols $\{*\}$ in Figs. 6(a)-(b). In this case, semiclassical open-orbit theory as formulated in [10] cannot give good results when the ejected electron energy comes near the resonance state. A semiclassical theory of such states must include reflections and tunneling, which are yet to be incorporated into the theory $[10,21]$. However, we see 

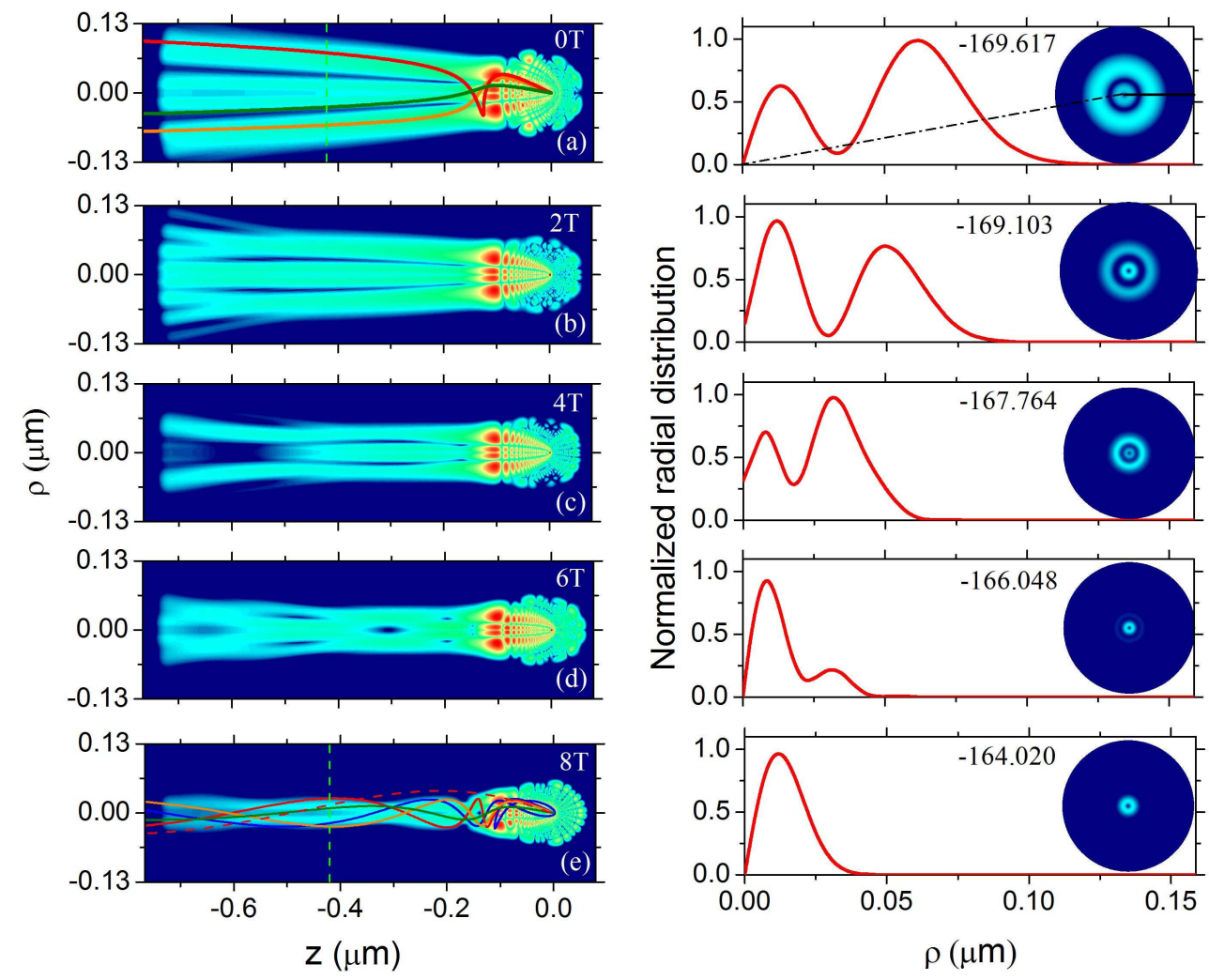

FIG. 5: (Color online) An overview of the calculated 2D spatial distributions of electron probability density, photoionization microscopy images, after a 500 ps evolution, and their normalized radial probability distributions on the detector for the red state $\left(\tilde{n}_{1}, \tilde{n}_{2}, m\right)=(1,28,0)$ in parallel fields with increasing magnetic field from $0 \mathrm{~T}$ to $8 \mathrm{~T}$, correspondingly shown in (a)-(e). The interference patterns on the detector plate shown on the right side are recorded at $z=-0.423 \mu \mathrm{m}(8000$ a.u.) and the detector positions are also shown by the green dashed line in (a) and (e). Several typical classical trajectories are shown by solid color lines for the cases of $B=0$ and $B=8 \mathrm{~T}$ in (a) and (e), respectively. The red dashed line in (e) is a typical trajectory with Coulomb interaction removed, indicating a non-neglectable focusing effect caused by Coulomb potential.
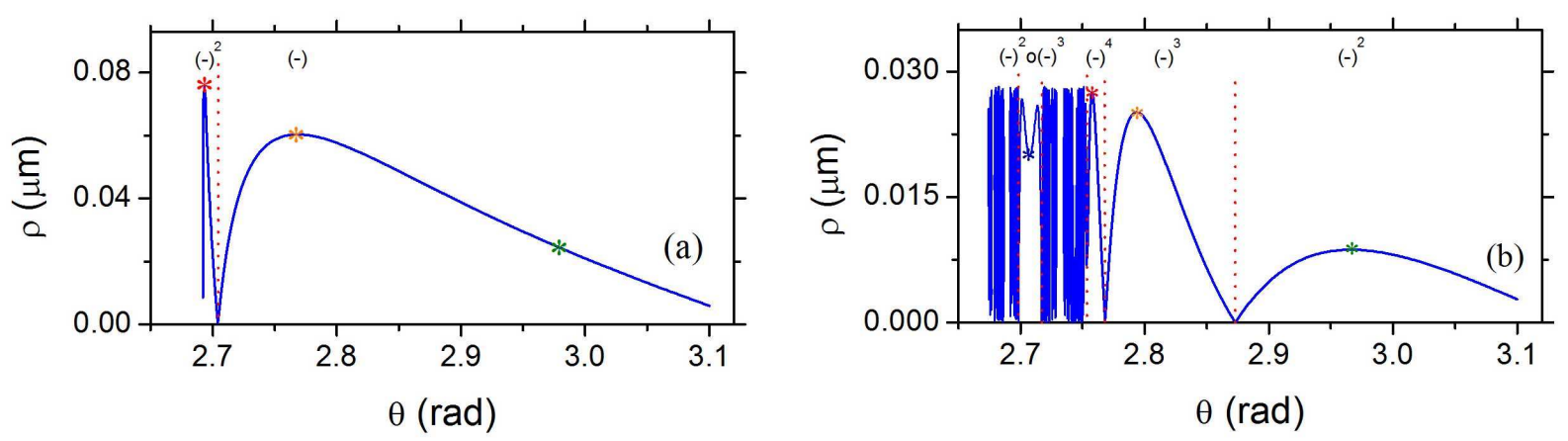

FIG. 6: (Color online) The dependence of the radius of impact on the detector on the initial angle $\theta$ at which the trajectory is launched. (a) $B=0 \mathrm{~T}$ and (b) $B=8 \mathrm{~T}$ correspond to results shown in Figs. 5 (a) and (e), respectively. The dashed lines connect each of escape segments to their corresponding patterns. Some "extremal" trajectories with $\partial \rho / \partial \theta_{i}=0$ at $z_{0}$ are marked by color symbols $\{*\}$, and the associated trajectories are shown with corresponding colors in Figs. 5(a) and (e). It should be noted that, in Figs. 6(a), the olive-colored $\{*\}$ corresponds to a trajectory that arrives at the outer edge of the inner circle in Figs. 5(a). 
that the boundaries of the classically allowed region coincide well with the extent of the quantum distribution.

Of course, the Coulomb interaction also plays an important role in the spatial electron probability distribution. In the absence of the Coulomb field, the photodetached electrons in parallel fields are associated with individual trajectories in spiral motion: harmonic oscillations in the radial $(\rho)$ direction, accompanied by uniform rotations in the azimuthal angle with a Larmor frequency $\omega_{L}=B / 2$. In the cases with and without Coulomb potential, the system energy satisfies $E=\frac{1}{2} v^{2}+V(r)$ in atomic units, where $V(r)=-1 / r+F z+B^{2} / 8 r^{2} \sin ^{2} \theta$ and $V(r)=F z+B^{2} / 8 r^{2} \sin ^{2} \theta$, respectively. With an $s$-wave approximation, each trajectory starts at initial velocity $v_{0}=\sqrt{2(E-V(r))}$ at a finite radius $a_{0}=50$ a.u. in all directions with the same weight [10]. A particular trajectory is illustrated in Fig. 5(e) for the case of parallel fields without the Coulomb interaction (red dashed line); it shares the same initial ejection angle theta $\theta=2.757$ with a corresponding trajectory including the Coulomb term (red solid line). Clearly, we see that the electron paths extend to larger $\rho$ when Coulomb interaction is absent. Thus, the Coulomb field causes focusing which reduces the maximum radial extension $\rho_{\max }$, and it also reduces the distance traveled by the electron between crossings of the $z$ axis, which are separated by one cyclotron time. In general, the interplay between the Coulomb interaction and parallel fields leads to more complex trajectories. Some of them become chaotic $[21,27]$, where a large number of trajectories encircle the nucleus many times. For comparison, a simple trajectory as depicted in blue solid line is given at an ejection angle $\theta=2.707$, which circles around the nucleus only once.

To observe the focusing effects of the magnetic field at the previous experimental condition of electric field of $F=808 \mathrm{~V} / \mathrm{cm}[3]$, we have to apply a strong magnetic field up to several Tesla. The focusing effects of the magnetic field are also presented for lower magnetic fields if we choose states close to the Stark saddle point at lower electric field as well, where the magnetic field induced interaction is comparable to that of electric field, for example, at $F=15 \mathrm{~V} / \mathrm{m}$ with the corresponding magnetic field is about $B=0.02 \mathrm{~T}$ [19], where the focusing effects are revealed in a space scale of tens of $\mu \mathrm{m}$.

\section{CONCLUSIONS}

We have performed theoretical calculations to study the dependence of the resonant energy positions, ejected electron spatial distributions and the dependence of their interference patterns on the magnetic field strength, for a hydrogen atom in parallel electric and magnetic fields. Red and blue states in a pure electric field are coupled to each other by the magnetic field. Owning to the quadratic diamagnetic term in the Hamiltonian, their energy maps show rich structures of anti-crossings. Red states occupy larger spatial volumes where electrons are more easily affected by the applied magnetic field in comparison to the more tightly confined blue states. In contrast to the case of a pure electric field, the number of dark fringes on the detector does not directly reveal the transverse nodal structure of resonances in parallel fields, particularly at stronger magnetic field.

\section{Acknowledgments}

This work is supported by the MOST 2013CB922003 and 2013CB922004 of the National Key Basic Research Program of China, and by NSFC (No. 91121005, No. 91421305, No. 11374176, No. 11274195 and No. 11174329), and by the National Science Foundation of the US under grant number 1404372.
[1] C. Blondel, C. Delsart, and F. Dulieu, Phys. Rev. Lett. 77, 3755 (1996).

[2] C. Nicole, H. L. Offerhaus, M. J. J. Vrakking, F. Lépine, and C. Bordas, Phys. Rev. Lett. 88, 133001 (2002).

[3] A. S. Stodolna, A. Rouzée, F. Lépine, S. Cohen, F. Robicheaux, A. Gijsbertsen, J. H. Jungmann, C. Bordas, and M. J. J. Vrakking, Phys. Rev. Lett. 110, 213001 (2013).

[4] S. Cohen, M. M. Harb, A. Ollagnier, F. Robicheaux, M. J. J. Vrakking, T. Barillot, F. Lépine, and C. Bordas, Phys. Rev. Lett. 110, 183001 (2013).

[5] V. D. Kondratovich and V. N. Ostrovsky, J. Phys. B 17, 1981 (1984).

[6] V. D. Kondratovich and V. N. Ostrovsky, J. Phys. B 17, 2011 (1984).

[7] V. D. Kondratovich and V. N. Ostrovsky, J. Phys. B 23, 3785 (1990).

[8] M. L. Du, Phys. Rev. A 40, 4983 (1989).
[9] C. Bordas, F. Lépine, C. Nicole, and M. J. J. Vrakking, Phys. Rev. A 68, 012709 (2003).

[10] L. B. Zhao and J. B. Delos, Phys. Rev. A 81, 053417 (2010).

[11] C. Nicole, I. Sluimer, F. Rosca-Pruna, M. Warntjes, M. Vrakking, C. Bordas, F. Texier, and F. Robicheaux, Phys. Rev. Lett. 85, 4024 (2000).

[12] F. Lepine, C. Bordas, C. Nicole, and M. J. J. Vrakking, Phys. Rev. A 70, 033417 (2004).

[13] F. Robicheaux and J. Shaw, Phys. Rev. Lett. 77, 4154 (1996).

[14] D. A. Harmin, Phys. Rev. A 24, 2491 (1981).

[15] L. B. Zhao, I. I. Fabrikant, J. B. Delos, F. Lépine, S. Cohen, and C. Bordas, Phys. Rev. A 85, 053421 (2012).

[16] L. B. Zhao and J. B. Delos, Phys. Rev. A 81, 053418 (2010).

[17] T. Kramer, C. Bracher, and M. Kleber, Europhys. Lett. 56, 471 (2001). 
[18] C. Bracher, T. Kramer, and J. B. Delos, Phys. Rev. A 73, 062114 (2006).

[19] C. Bracher and J. B. Delos, Phys. Rev. Lett. 96, 100404 (2006).

[20] D. Wang, Eur. Phys. J. D 45, 179 (2007).

[21] L. Wang, H. F. Yang, X. J. Liu, H. P. Liu, M. S. Zhan, and J. B. Delos, Phys. Rev. A 82, 022514 (2010).

[22] M. Courtney, Phys. Rev. A 51, 4558 (1995).

[23] M. A. Iken, F. Borondo, R. M. Benito, and T. Uzer, Phys. Rev. A 49, 2734 (1994).
[24] P. Cacciani, E. Luc-Koenig, J. Pinard, C. Thomas, and S. Liberman, J. Phys. B: At. Mol. Opt. Phys. 21, 3499 (1988).

[25] W. Gao, H. F. Yang, H. Cheng, X. J. Liu, and H. P. Liu, Phys. Rev. A 86, 012517 (2012).

[26] L. B. Zhao, D. H. Xiao, and I. I. Fabrikant, Phys. Rev. A 91, 043405 (2015).

[27] K. A. Mitchell and J. B. Delos, Physica D 229, 9 (2007). 